\title{
On computation of the compositional rule of inference under triangular norms*
}

\author{
R. Fullér ${ }^{\dagger}$ \\ rfuller@abo.fi
}

\author{
H.-J.Zimmermann \\ zi@buggi.or.rwth-aachen.de
}

\begin{abstract}
This paper is devoted to the derivation of exact calculation formulas for the compositional rule of inference under Archimedean t-norms, when both the observation and the relation parts are given by Hellendoorn's $\phi$-function [6].
\end{abstract}

Keywords: Triangular norm, compositional rule of inference, Lagrange's multipliers method,

\section{Introduction}

The inference process from imprecise or vague premises is becoming more and more important for knowledge-based systems, especially for fuzzy expert systems $[9,12,13,15]$. In approximate reasoning there are several kinds of inference rules, which deal with the problem of deduction of conclusions in an imprecise setting. An important problem is the (approximate) computation of the membership function of the conclusion in these schemes $[1,4,6,7,8,10]$. This paper deals with the derivation of exact calculation formulas for the compositional rule of inference, which has the global scheme [14]

\begin{tabular}{ll} 
Observation: & $x$ has property $P$ \\
Relation: & $x$ and y have relation $W$ \\
\hline Conclusion: & $y$ has property $Q$
\end{tabular}

where the membership function of the conclusion $\mathrm{Q}$ is defined by a sup-t-norm composition of $P$ and $W$

$$
Q(y)=\sup \text { t-norm }(P(x), W(x, y))
$$

In [6] Hellendoorn showed the closure property of the compositional rule of inference under sup-min composition and presented exact calculation formulas for the membership function of the conclusion when both the observation and relation parts are given by S-, $\pi-$, or $\phi$-function. Our results are connected with those presented in [6] and we generalize them. We shall determine the exact membership function of the conclusion, when both the observation and the part of the relation (rule) are given by concave $\phi$-function [6]; and the t-norm is Archimedean with a strictly convex additive generator function. The efficiency of our method stems from the fact that the distributions, involved in the relation and observation, are represented by a parametrized $\phi$-function. The deduction process then consists of some simple computations performed on the parameters.

\footnotetext{
${ }^{*}$ The final version of this paper appeared in: R. Fullér and H.-J. Zimmermann On computation of the compositional rule of inference under triangular norms Fuzzy Sets and Systems, 51(1992) 267-275. doi: 10.1016/0165-0114(92)90017-X

${ }^{\dagger}$ Presently at Lehrstuhl für Unternehmensforschung, RWTH Aachen, Templergraben 64, W-5100 Aachen, Germany
} 


\section{Definitions}

A fuzzy interval $\tilde{a}$ is a fuzzy set of real numbers $\mathbb{R}$ with a continuous, compactly supported, unimodal and normalized membership function. It is known [2], that any fuzzy interval $\tilde{a}$ can be described by the following membership function:

$$
\tilde{a}(x)= \begin{cases}L\left(\frac{a-t}{\alpha}\right) & \text { if } t \in[a-\alpha, a] \\ 1 & \text { if } t \in[a, b] \\ R\left(\frac{t-b)}{\beta}\right) & \text { if } t \in[b, b+\beta] \\ 0 & \text { otherwise }\end{cases}
$$

where $[a, b]$ is the peak of $\tilde{a} ; a$ and $b$ are the lower and upper modal values; $L$ and $R$ are shape functions: $[0,1] \rightarrow[0,1]$, with $L(0)=R(0)=1$ and $L(1)=R(1)=0$ which are non-increasing, continuous mappings. These fuzzy intervals are called of type L-R and denoted by $\tilde{a}=(a, b, \alpha, \beta)_{L R}$. The support of $\tilde{a}$ is $[a-\alpha, b+\beta]$. A function $T:[0,1] \times[0,1] \rightarrow[0,1]$ is said to be a triangular norm [11] (t-norm for short) iff $T$ is symmetric, associative,non-decreasing in each argument, and $T(x, 1)=x$ for all $x \in[0,1]$. Recall that a t-norm $T$ is Archimedean iff $T$ is continuous and $T(x, x)<x$ for all $x \in(0,1)$. Every Archimedean t-norm $T$ is representable by a continuous and decreasing function $f:[0,1] \rightarrow[0, \infty]$ with $f(1)=0$ and

$$
T(x, y)=f^{[-1]}(f(x)+f(y))
$$

where $f^{[-1]}$ is the pseudo-inverse of $f$, defined by

$$
f^{[-1]}(y)= \begin{cases}f^{-1}(y) & \text { if } y \in[0, f(0)] \\ 0 & \text { otherwise }\end{cases}
$$

The function $\mathrm{f}$ is the additive generator of $T$. If $T$ is a t-norm and $\tilde{a}_{1}, \tilde{a}_{2}$ are fuzzy sets of the real line (i.e. fuzzy quantities) then their $T$-sum $\tilde{A}:=\tilde{a}_{1}+\tilde{a}_{2}$ is defined by

$$
\tilde{A}(z)=\sup _{x_{1}+x_{2}=z} T\left(\tilde{a}_{1}\left(x_{1}\right), \tilde{a}_{2}\left(x_{2}\right)\right), z \in \mathbb{R}
$$

which can be written in the form

$$
\tilde{A}(z)=f^{[-1]}\left(f\left(\tilde{a}_{1}\left(x_{1}\right)\right)+f\left(\tilde{a}_{2}\left(x_{2}\right)\right)\right)
$$

supposing that $f$ is the additive generator of $T$.

Since $f$ is continuous and decreasing, $f^{[-1]}$ is also continuous and non-increasing, we have

$$
\tilde{A}(z)=f^{[-1]}\left(\inf _{x_{1}+x_{2}=z}\left(f\left(\tilde{a}_{1}\left(x_{1}\right)\right)+f\left(\tilde{a}_{2}\left(x_{2}\right)\right)\right) .\right.
$$

\section{Auxiliary lemma}

Lemma 3.1 Let T be an Archimedean t-norm with additive generator $f$ and let $\tilde{a}_{i}=\left(a_{i}, b_{i}, \alpha, \beta\right)_{L R}, i=$ 1,2 be fuzzy intervals of type $L-R$. If $L$ and $R$ are twice differentiable, concave functions, and $f$ is a twice 
differentiable, strictly convex function, then the membership function of their T-sum $\tilde{A}=\tilde{a}_{1}+\tilde{a}_{2}$ is

$$
\tilde{A}_{2}(z)= \begin{cases}1 & \text { if } A \leq z \leq B \\ f^{[-1]}\left(2 \times f\left(L\left(\frac{A-z}{2 \alpha}\right)\right)\right) & \text { if } A-2 \alpha \leq z \leq A \\ f^{[-1]}\left(2 \times f\left(R\left(\frac{z-B}{2 \beta}\right)\right)\right) & \text { if } B \leq z \leq B+2 \beta \\ 0 & \text { otherwise }\end{cases}
$$

where $A=a_{1}+a_{2}$ and $B=b_{1}+b_{2}$.

Proof. As mentioned above, the investigated membership function is

$$
\tilde{A}(z)=f^{[-1]}\left(\inf _{x_{1}+x_{2}=z}\left(f\left(\tilde{a}_{1}\left(x_{1}\right)\right)+f\left(\tilde{a}_{2}\left(x_{2}\right)\right)\right)\right) .
$$

It is easy to see that the support of $\tilde{A}$ is included in the interval $[A-2 \alpha, B+2 \beta]$. From the decomposition rule of fuzzy intervals into two separate parts [3] it follows that the peak of $\tilde{A}$ is $[A, B]$. Moreover, if we consider the right hand side of $\tilde{A}$ (i.e. $\{z: \leq z \leq B+2 \beta\}$ ) then only the right hand sides of terms $\tilde{a}_{1}$ and $\tilde{a}_{2}$ become relevant in (1) (i.e. $b_{i} \leq x_{i} \leq b_{i}+\beta, i=1,2$ ). The same holds for the left hand side of $\tilde{A}$ (i.e. if $A-2 \alpha \leq z \leq A$ then $\left.a_{i}-\alpha \leq x_{i} \leq a_{i}, i=1,2\right)$. Let us now consider the right hand side of $\tilde{A}$, so let $B \leq z$ leqB $+2 \beta$. (A similar method can be used for the computation of $\tilde{A}(z)$, if $z \in[A-2 \alpha, A]$.$) The constraints$

$$
x_{1}+x_{2}=z, \quad b_{i} \leq x_{i} \leq b_{i}+\beta, i=1,2,
$$

determine a compact and convex domain $\mathcal{K} \subset \mathbb{R}^{2}$ which is the intersection of the rectangle,

$$
\mathcal{B}:=\left\{\left(x_{1}, x_{2}\right) \in \mathbb{R}^{2} \mid b_{i} \leq x_{i} \leq b_{i}+\beta\right\}, i=1,2,
$$

and the hyperplane

$$
\mathcal{P}:=\left\{\left(x_{1}, x_{2}\right) \in \mathbb{R}^{2} \mid x_{1}+x_{2}=z\right\} .
$$

In order to determine $\tilde{A}(z)$, we have to solve the following mathematical programming problem:

$$
\phi\left(x_{1}, x_{2}\right):=f\left(\tilde{a}_{1}\left(x_{1}\right)\right)+f\left(\tilde{a}_{2}\left(x_{2}\right)\right) ; \quad \text { subject to }\left(x_{1}, x_{2}\right) \in \mathcal{K} .
$$

Since $\mathcal{K}$ is compact and $\phi$ is continuous, the infinum can be changed to minimum.

(for the complete proof see: Fuzzy Sets and Systems, 51(1992) 267-275)

It should be noted that Lemma 1 can be extended to the case of $n$ fuzzy intervals [5].

\section{Computation of the compositional rule of inference}

Following [6] we use the $\phi$-function for the representation of linguistic notions

$$
\phi(x ; a, b, c, d)= \begin{cases}1 & \text { if } b \leq x \leq c \\ \phi_{1}\left(\frac{x-a}{b-c}\right) & \text { if } a \leq x \leq b, a<b, \\ \phi_{2}\left(\frac{x-c}{d-c}\right) & \text { if } c \leq x \leq d, c<d, \\ 0 & \text { otherwise }\end{cases}
$$


where $\phi_{1}:[0,1] \rightarrow[0,1]$ is continuous, monoton increasing function and $\phi_{1}(0)=0, \phi_{1}(1)=1$; $\phi_{2}:[0,1] \rightarrow[0,1]$ is continuous, monoton decreasing function and $\phi_{2}(0)=1, \phi_{2}(1)=0$ So $\phi$ is a function which is 0 left of $a$, increases to 1 in $(a, b)$, is 1 in $[b, c]$, decreases to 0 in $(c, d)$ and is 0 right of $d$ (for the sake of simplicity, we do not consider the cases $a=b$ or $c=d$ ). It should be noted that $\phi$ can be considered as the membership function of the fuzzy interval $\tilde{a}=(b, c, b-a, d-c)_{L R}$, with $R(x)=\phi_{2}(x)$ and $L(x)=\phi_{1}(1-x)$.

We consider the compositional rule of inference,

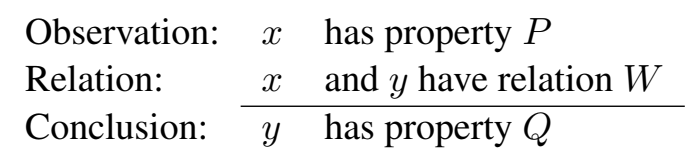

where, the membership functions of $P$ and $W$ are defined by means of a particular $\phi$-function, and the membership function of the conclusion $Q$ is defined by sup- $T$ composition of $P$ and $W$

$$
Q(y)=\left(P \circ_{T} W\right)(y)=\sup _{x} T(P(x), W(x, y)) .
$$

The following theorem presents an efficient method for the exact computation of the membership function of the conclusion.

Theorem 4.1 Let $T$ be an Archimedean t-norm with additive generator $f$ and let $P(x)=\phi(x ; a, b, c, d)$ and $W(x, y)=\phi(y-x ; a+u, b+u, c+v, d+v)$. If $\phi_{1}$ and $\phi_{2}$ are twice differentiable, concave functions, and $f$ is a twice differentiable, strictly convex function, then

$$
Q(y)= \begin{cases}1 & \text { if } 2 b+u \leq y \leq 2 c+v \\ f^{[-1]}\left(2 f\left(\phi_{1}\left[\frac{y-2 a-u}{2(b-a)}\right]\right)\right) & \text { if } 2 a+u \leq y \leq 2 b+u \\ f^{[-1]}\left(2 f\left(\phi_{2}\left[\frac{y-2 c-v}{2(d-c)}\right]\right)\right) & \text { if } 2 c+v \leq y \leq 2 d+v \\ 0 & \text { otherwise }\end{cases}
$$

Proof. (see Fuzzy Sets and Systems, 51(1992) 267-275).

Remark 4.1 It should be noted that we have calculated the membership function of $Q$ under the assumption that the left and right spreads of $P$ do not differ from the left and right spreads of $W$ (the lengths of their tops can be different). To determine the exact membership function of $Q$ in the general case: $P(x)=\phi\left(x ; a_{1}, a_{2}, a_{3}, a_{4}\right)$ and $W(x, y)=\phi\left(y-x ; b_{1}, b_{2}, b_{3}, b_{4}\right)$ seems to be very difficult and it is possible, that there does not exist an explicit formula for it.

\section{Applications}

Using Theorem 1 we compute the exact membership function of the conclusion $Q$ in the case of Yager's, Dombi's and Hamacher's parametrized t-norm. Let us consider the following scheme

$$
\begin{array}{ll}
P(x) & =\phi(x ; a, b, c, d) \\
W(y, x) & =\phi(y-x ; a+u, b+u, c+v, d+v) \\
Q(y) & =
\end{array}
$$




\section{Denoting}

$$
\sigma:=\frac{(y-2 a-u)}{2(b-a)}, \quad \theta:=\frac{y-2 c-v}{2(d-c)},
$$

we get the following formulas for the membership function of the conclusion $Q$.

(i) Yager's t-norm with $p>1$. Here

$$
T(x, y)=1-\min \left\{1, \sqrt[p]{(1-x)^{p}+(1-y)^{p}}\right\} .
$$

with generator $f(t)=(1-t)^{p}$, and

$$
Q(y)= \begin{cases}1-2^{1 / p}\left(1-\phi_{1}(\sigma)\right) & \text { if } 0<\sigma<\phi_{1}^{-1}\left(2^{-1 / p}\right) \\ 1 & \text { if } 2 b+u \leq y \leq 2 c+v \\ 1-2^{1 / p}\left(1-\phi_{2}(\theta)\right) & \text { if } 0<\theta<\phi_{2}^{-1}\left(1-2^{1 / p}\right)\end{cases}
$$

(ii) Hamacher's t-norm with $p \leq 2$. Here

$$
T(x, y)=\frac{x y}{p+(1-p)(x+y-x y)}
$$

with generator

$$
f(t)=\ln \frac{p+(1-p) t}{t}
$$

and

$$
Q(y)= \begin{cases}p /\left(\tau_{1}^{2}-1+p\right) & \text { if } 0<\sigma<1 \\ 1 & \text { if } 2 b+u \leq y \leq 2 c+v \\ p /\left(\tau_{2}^{2}-1+p\right) & \text { if } 0<\theta<1\end{cases}
$$

where

$$
\tau_{1}=\frac{p+(1-p) \phi_{1}(\sigma)}{\phi_{1}(\sigma)}, \quad \tau_{2}=\frac{p+(1-p) \phi_{2}(\sigma)}{\phi_{2}(\sigma)}
$$

(iii) Dombi's t-norm with $p>1$. Here

$$
T(x, y)=\frac{1}{1+\sqrt[p]{(1 / x-1)^{p}+(1 / y-1)^{p}}}
$$

with additive generator

$$
f(t)=\left(\frac{1}{t}-1\right)^{p}
$$

and

$$
Q(y)= \begin{cases}1 /\left(1+2^{1 / p} \tau_{3}\right) & \text { if } 0<\sigma<1 \\ 1 & \text { if } 2 b+u \leq y \leq 2 c+v \\ 1 /\left(1+2^{1 / p} \tau_{4}\right) & \text { if } 0<\theta<1\end{cases}
$$

where

$$
\tau_{3}=\frac{1}{\phi_{1}(\sigma)}-1, \quad \tau_{4}=\frac{1}{\phi_{2}(\sigma)}-1
$$

Example 1 We illustrate Theorem 1 by the following example: 


\begin{tabular}{ll}
$x$ is close to 3 & $\phi(x ; 1,3,4,7)$ \\
$x$ and $y$ are approximately equal & $\phi(y-x ;-2,0,0,3)$ \\
\hline$y$ is more or less close to $[3,4]$ & $Q(y)$
\end{tabular}

where

$$
Q(y)= \begin{cases}1 & \text { if } 2 b+u \leq y \leq 2 c+v \\ f^{[-1]}\left(2 f\left(\phi_{1}\left[\frac{y-2 a-u}{2(b-a)}\right]\right)\right) & \text { if } 2 a+u \leq y \leq 2 b+u \\ f^{[-1]}\left(2 f\left(\phi_{2}\left[\frac{y-2 c-v}{2(d-c)}\right]\right)\right) & \text { if } 2 c+v \leq y \leq 2 d+v \\ 0 & \text { otherwise }\end{cases}
$$

We have used the membership function $\phi(y-x ;-2,0,0,3)$ to describe " $x$ and $y$ are approximately equal". This means that the membership degree is one, iff $x$ and $\mathrm{y}$ are equal in the classical sense. If $y-x>2$ or $x-y>3$, then the degree of membership is 0 . The conclusion $Q$ has been called " $y$ is more or less close to $[3,4]$ ", because $P(t)=Q(T)=1$, when $t \in[3,4]$ and $P(t)<Q(t)$ otherwise.

\section{References}

[1] R.Da, A critical study of widely used fuzzy implication operators and their influence on the inference rules in fuzzy expert systems, Ph.D. Thesis, State University of Ghent, 1990.

[2] D.Dubois and H.Prade, Fuzzy Sets and Systems: Theory and Applications, Academic Press, New York, 1980.

[3] D.Dubois and H.Prade, Additions of interactive fuzzy numbers, IEEE Transactions on Automatic Control,1981, Vol. AC-26, No.4 926-936.

[4] D.Dubois, R.Martin-Clouarie and H.Prade, Practical computing in fuzzy logic, in: M.M.Gupta and T.Yamakawa eds., Fuzzy Computing: Theory, Hardware, and Applications, North-Holland, Amsterdam, 1988 11-34.

[5] R.Fullér and T.Keresztfalvi, t-norm-based addition of fuzzy intervals, BUSEFAL, 46(1991) 27-32.

[6] H.Hellendoorn, Closure properties of the compositional rule of inference, Fuzzy Sets and Systems, 35(1990) 163-183.

[7] H.Hellendoorn, The generalized modus ponens considered as a fuzzy relation, Fuzzy Sets and Systems, (to appear).

[8] R.Martin-Clouarie, Semantics and computation of the generalized modus ponens: The long paper, International Journal of Approximate Reasoning, 3(1989) 195-217.

[9] P.Margrez, P.Smets, Fuzzy modus ponens: A new model suitable for applications in knowledgebased systems, International Journal of Intelligent Systems, 4(1989) 181-200.

[10] M.Mizumoto and H.-J.Zimmermann, Comparison of fuzzy reasoning methods, Fuzzy Sets and Systems, 8(1982) 253-283.

[11] B.Schweizer, A.Slkar, Associative functions and abstract semigroups, Publ. Math. Debrecen, 10(1963) 69-81. 
[12] R.R.Yager, Approximate reasoning as a basis for rule-based expert systems, IEEE Transactions on Systems, Man, and Cybernetics, 14(1984) 636-643.

[13] L.A.Zadeh, The role of fuzzy logic in the management of uncertainty in expert systems, Fuzzy Sets and Systems, 11(1983) 199-228.

[14] L.A.Zadeh, The concept of linguistic variable and its applications to approximate reasoning, Parts I,II,III, Information Sciences, 8(1975) 199-251; 8(1975) 301-357; 9(1975) 43-80.

[15] H.-J.Zimmermann, Fuzzy sets, Decision Making and Expert Systems, Boston, Dordrecht, Lancaster 1987.

\section{Follow ups}

The results of this paper have been improved and/or generalized in the following works.

\section{in journals}

A26-c26 M Štepnicka, B Jayaram, On the Suitability of the Bandler-Kohout Subproduct as an Inference Mechanism, IEEE TRANSACTIONS ON FUZZY SYSTEMS, 18(2010), number 2, pp. 285-298. 2010

http://dx.doi.org/10.1109/TFUZZ.2010.2041007

There are many works that have proposed modifications to the classical CRI in an attempt to enhance the efficiency in its inferencing (see, for example, the works of Fullér and coauthors [48]-[50] and Moser and Navara [51]-[53]). (page 295)

A26-c25 B. Jayaram, On the Law of Importation $(x \wedge y) \longrightarrow z \equiv(x \longrightarrow(y \longrightarrow z))$ in Fuzzy Logic, IEEE Transactions on Fuzzy Systems, vol.16, no.1, pp.130-144, Feb. 2008

http://dx.doi.org/10.1109/TFUZZ.2007.895969

A26-c24 Hong DH, Hwang C, Kim KT T-sum of sigmoid-shaped fuzzy intervals INFORMATION SCIENCES, 177 (18): 3831-3839 SEP 152007

http://dx.doi.org/10.1016/j.ins.2007.03.023

A26-c23 Hong DH, T-sum of bell-shaped fuzzy intervals, FUZZY SETS AND SYSTEMS, 158 (7): 739-746 APR 12007

http://dx.doi.org/10.1016/j.fss.2006.10.021

A26-c22 Hong DH On types of fuzzy numbers under addition KYBERNETIKA, 40 (4): 469-476 2004

http://kybernetika.utia.cas.cz/pdf_article/40_4_654_full.pdf

A26-c21 Dug Hun Hong, T-sum of L-R fuzzy numbers with unbounded supports, Commun. Korean Math. Soc., 18 (2003), no. 2, 385-392. 2003

A26-c20 Hong DH, On shape-preserving additions of fuzzy intervals, JOURNAL OF MATHEMATICAL ANALYSIS AND APPLICATIONS, 267 (1): 369-376, MAR 12002 
A26-c19 Dug Hun Hong, Some results on the addition of fuzzy intervals FUZZY SETS AND SYSTEMS, 122(2001) 349-352. 2001

http://dx.doi.org/10.1016/S0165-0114(00)00005-1

A26-c18 Seok Yoon Hwang and Hyo Sam Lee, Nilpotent t-norm-based sum of fuzzy intervals, FUZZY SETS AND SYSTEMS, 123(2001) 73-80. 2001

http://dx.doi.org/10.1016/S0165-0114(01)00005-7

In particular if $\alpha_{1}=\alpha_{2}, \beta_{1}=\beta_{2}$ then $\tilde{A}^{*}=\tilde{A}_{1} \oplus_{T} \tilde{A}_{2}$, which generalizes the results by Fullér and Zimmermann [A24, A26]. (page 74)

A26-c17 Dug Hun Hong Shape preserving multiplications of fuzzy numbers, FUZZY SETS AND SYSTEMS, 123(2001) 81-84. 2001

http://dx.doi.org/10.1016/S0165-0114(00)00107-X

A26-c16 D.H.Hong and C. Hwang, A T-sum bound of LR-fuzzy numbers, FUZZY SETS AND SYSTEMS, 91(1997) 239-252. 1997

http://dx.doi.org/10.1016/S0165-0114(97)00144-9

Now, in the case of different spreads, we are naturally asked about how to determine the exact membership function. Fullér and Zimmermann [A26] mentioned in their remark 1 that it seems to be very difficult and complicated to determine the exact membership function of $T$-sum of LR-fuzzy numbers. (page 241)

We also consider the compositional rule of inference under triangular norms stated by Fullér and Zimmermann [A26]. (page 252)

A26-c15 A.Markova, T-sum of L-R fuzzy numbers, FUZZY SETS AND SYSTEMS, 85(1997) 379384. 1997

http://dx.doi.org/10.1016/0165-0114(95)00370-3

Using our results, we guarantee the validity of Fullér-Zimmermann composition law for $f \circ \phi_{1}$ and $f \circ \phi_{2}$ convex ... (page 383)

A26-c14 R.Mesiar, Shape preserving additions of fuzzy intervals, FUZZY SETS AND SYSTEMS, 86(1997) 73-78. 1997

http://dx.doi.org/10.1016/0165-0114(95)00401-7

Some applications of above results are expected also in the domain of compositional rules of inference under nilpotent t-norms, see e.g. [A26]. (page 78)

A26-c13 A.Markova, Addition of L-R fuzzy numbers, Bulletin for Studies and Exchanges on Fuzziness and its Applications, 63(1995) 25-29. 1995

A26-c12 D.H.Hong and S.Y.Hwang, On the compositional rule of inference under triangular norms, FUZZY SETS AND SYSTEMS, 66(1994) 25-38. 1994

http://dx.doi.org/10.1016/0165-0114(94)90299-2 
The aim of this paper is to provide a close upper bound of the membership function for the compositional rule of inference under Archimedean t-norm, where both the observation and the relation parts are given by Hellendoorn's $\phi$-function (1980). In particular, if the left and right spreads of the observation part is the same as those of the relation part, then this upper bound is the exact membership function, which generalizes the earlier results by Fullér and Zimmermann (1992) in that the assumption of twice differentiability is deleted. (page 25)

Fullér and Zimmermann [A26] wrote a paper which deals with the derivation of exact calculation formulas for the compositional rule of inference, which has the global scheme [7]

$$
\begin{array}{lll}
\text { Observation: } & x & \text { has property } P \\
\text { Relation: } & x & \text { and } y \text { have relation } W \\
\text { Conclusion: } & y & \text { has property } Q
\end{array}
$$

where the membership function of the conclusion $Q$ is defined by sup-t-norm composition of $P$ and $W$ :

$$
Q(y)=\sup _{x} \mathrm{t} \text {-norm }(P(x), W(x, y)) .
$$

(page 26)

In [6] Hellendoorn showed the closure property of the compositional rule of inference under sup-min-norm composition and prsented exact calculation formulas for the membership function of the conclusion when both the observation and relation parts are given by $S-, \pi-$, or $\phi$-functions. Fullér and Zimmermann's results are connected with those presented in [6] and they generalize them as follows:

Theorem 1.1 [A26] Let $T$ be an Archimedean t-norm with additive generator $f$ and let $P(x)=\phi(x ; a, b, c, d)$ and $W(x, y)=\phi(y-x ; a+u, b+u, c+v, d+v)$. If $\phi_{1}$ and $\phi_{2}$ are twice differentiable, concave functions, and $f$ is a twice differentiable, strictly convex function, then

$$
Q(y)= \begin{cases}1 & \text { if } 2 b+u \leq y \leq 2 c+v \\ f^{[-1]}\left(2 f\left(\phi_{1}\left(\frac{y-2 a-u}{2(b-a)}\right)\right)\right) & \text { if } 2 a+u \leq y \leq 2 b+u \\ f^{[-1]}\left(2 f\left(\phi_{2}\left(\frac{y-2 c-v}{2(d-c)}\right)\right)\right) & \text { if } 2 c+v \leq y \leq 2 d+v \\ 0 & \text { otherwise. }\end{cases}
$$

(page 26)

From Theorem 2.4 we know that if the left and right spreads of $P$ are equal to the left and right spreads of $W$, respectively, then the exact membership function of the conlusion $Q$ can be determined without the condition of differentiability of $\phi_{1}, \phi_{2}$ and $f$ in Fullér and Zimmermann's theorem [A26]. (page 29)

\section{in proceedings and in edited volumes}


A26-c9 M.Štepnicka and B. Jayaram, On Computational Aspects of the BK-Subproduct Inference Mechanism, 18th IEEE International Conference on Fuzzy Systems, FUZZ-IEEE 2009, Jeju Island, Korea, August 20-24, 2009, pp. 1181-1186. 2009

http://dx.doi.org/10.1109/FUZZY.2009.5277076

A26-c9 Inma P. Cabrera, Pablo Cordero, and Manuel Ojeda-Aciego, Fuzzy Logic, Soft Computing, and Applications, in: Joan Cabestany; Francisco Sandoval; Alberto Prieto; Juan M. Corchado eds., Bio-Inspired Systems: Computational and Ambient Intelligence, Lecture Notes in Computer Science, vol. 5517/2009, Springer, [ISBN 978-3-642-02477-1], 2009, pp. 236-244. 2009

http://dx.doi.org/10.1007/978-3-642-02478-8_30

A26-c8 D.Dubois, E.Kerre, R.Mesiar and H.Prade, Fuzzy interval analysis, in: Didier Dubois and Henri Prade eds., Fundamentals of Fuzzy Sets, The Handbooks of Fuzzy Sets Series, Volume 7, Kluwer Academic Publishers, [ISBN 0-7923-7732-X], 2000, 483-581. 2000

A26-c7 D.H.Hong and C. Hwang, Upper bound of T-sum of LR-fuzzy numbers, in: Proc. of IPMU'96 Conference (July 1-5, 1996, Granada, Spain), 1996 343-346. 1996

\section{in books}

A26-c6 Da Ruan, Etienne E. Kerre, Fuzzy If-then Rules in Computational Intelligence: Theory and Applications, Kluwer, [ISBN 9780792378204], 2000.

A26-c5 E. P. Klement, Radko Mesiar, Endre Pap, Triangular Norms, Springer, [ISBN 978-0-79236416-0], 2000.

http://www.springer.com/philosophy/logic+and+philosophy+of+language/book/978-0-7923-64160

A26-c4 Jonathan S. Golan, Semirings and Their Applications, Springer, [ISBN: 978-0-7923-5786-5], 1999.

http://www.springer.com/mathematics/algebra/book/978-0-7923-5786-5

A26-c3 Jonathan S. Golan, Power Algebras over Semirings, Springer, [ISBN 978-0-7923-5834-3], 1999.

http://www.springer.com/mathematics/algebra/book/978-0-7923-5834-3

A26-c2 R. Lowen, Fuzzy Set Theory, Kluwer Academic Publishers, [ISBN: 0-7923-4057-4], 1996.

A26-c1 G.J.Klir and B.Yuan, Fuzzy Sets and Fuzzy Logic: Theory and Applications, Prentice Hall, [ISBN 0-13-101171-5], 1995. 